\title{
SGLT2 Inhibitors, What the Emergency Physician Needs to Know: A Narrative Review
}

\author{
Henri Lu ${ }^{1, *(D)}$, Hortense Lu ${ }^{2}$, Christophe Kosinski ${ }^{3}{ }^{(D}$, Anne Wojtusciszyn ${ }^{3}$, Anne Zanchi ${ }^{3}$, \\ Pierre-Nicolas Carron ${ }^{4}$, Martin Müller ${ }^{5}{ }^{\mathbb{D}}$, Philippe Meyer ${ }^{6}$, Jehan Martin ${ }^{7}$, Olivier Muller ${ }^{1}$ and Roger Hullin ${ }^{1}$
}

1 Service of Cardiology, Cardiovascular Department, Lausanne University Hospital, 1011 Lausanne, Switzerland; olivier.muller@CHUV.ch (O.M.); Roger.Hullin@chuv.ch (R.H.)

2 Emergency Department, Saint-Joseph Hospital, 75014 Paris, France; lu.hortense@gmail.com

3 Service of Endocrinology, Diabetes and Metabolism, Lausanne University Hospital, 1011 Lausanne, Switzerland; Christophe.Kosinski@chuv.ch (C.K.); Anne.Wojtusciszyn@chuv.ch (A.W.); Anne.Zanchi@chuv.ch (A.Z.)

4 Emergency Department, Lausanne University Hospital, 1011 Lausanne, Switzerland; Pierre-Nicolas.Carron@chuv.ch

5 Emergency Department, Bern University Hospital, 3010 Bern, Switzerland; martin.mueller2@insel.ch 6 Cardiology Service, Geneva University Hospitals, 1205 Geneva, Switzerland; Philippe.meyer@hcuge.ch

7 Emergency Department, Geneva University Hospital, 1205 Geneva, Switzerland; jehan.martin@hcuge.ch

* Correspondence: henri.lu@chuv.ch; Tel.: +41-79-556-03-89

Citation: Lu, H.; Lu, H.; Kosinski, C.; Wojtusciszyn, A.; Zanchi, A.; Carron, P.-N.; Müller, M.; Meyer, P.; Martin, J.; Muller, O.; et al. SGLT2 Inhibitors, What the Emergency Physician Needs to Know: A Narrative Review. J. Clin. Med. 2021, 10, 2036. https://doi.org/ $10.3390 / \mathrm{jcm} 10092036$

Academic Editor: Giorgio Costantino

Received: 1 April 2021

Accepted: 7 May 2021

Published: 10 May 2021

Publisher's Note: MDPI stays neutral with regard to jurisdictional claims in published maps and institutional affiliations.

Copyright: (c) 2021 by the authors. Licensee MDPI, Basel, Switzerland. This article is an open access article distributed under the terms and conditions of the Creative Commons Attribution (CC BY) license (https:/ / creativecommons.org/licenses/by/ $4.0 /)$.

\begin{abstract}
Canagliflozin, dapagliflozin, empagliflozin, and ertugliflozin belong to a class of antidiabetic treatments referred to as sodium-glucose cotransporter 2 inhibitors (SGLT2 inhibitors, or SGLT2is). SGLT2is are currently indicated in North America and in Europe in type 2 diabetes mellitus, especially in patients with cardiovascular (CV) disease, high CV risk, heart failure, or renal disease. In Europe, dapagliflozin is also approved as an adjunct to insulin in patients with type 1 diabetes mellitus. New data provide evidence for benefits in heart failure with reduced ejection fraction and chronic kidney disease, including in patients without diabetes. The use of SGLT2is is expected to increase, suggesting that a growing number of patients will present to the emergency departments with these drugs. Most common adverse events are easily treatable, including mild genitourinary infections and conditions related to volume depletion. However, attention must be paid to some potentially serious adverse events, such as hypoglycemia (when combined with insulin or insulin secretagogues), lower limb ischemia, and diabetic ketoacidosis. We provide an up-to-date practical guide highlighting important elements on the adverse effects of SGLT2is and their handling in some frequently encountered clinical situations such as acute heart failure and decompensated diabetes.
\end{abstract}

Keywords: diabetes; heart failure; renal; guidelines

\section{Introduction}

Sodium glucose-cotransporter 2 inhibitors (SGLT2is) are a class of oral antihyperglycemic agents that block glucose and sodium reabsorption in the proximal tubule of the kidney, causing glucosuria and osmotic diuresis. They improve glycemic control in patients with type 2 diabetes mellitus (T2DM) and provide cardiovascular (CV) and renal benefits independently of diabetes status [1-3].

Canagliflozin, dapagliflozin, empagliflozin, and ertugliflozin are indicated as first- or second-line treatments in T2DM individuals with CV disease, high CV risk, heart failure, and chronic kidney disease (CKD) both in Europe and North America, provided that in some European countries, their use may be prohibited if kidney clearance is below $45 \mathrm{~mL} / \mathrm{min}[4,5]$. Dapagliflozin is also indicated in patients with heart failure with reduced ejection fraction (HFrEF) independently of diabetes status and, in Europe, as an adjunct to insulin in T1DM [6,7]. Despite an overall favorable safety profile, a few side effects of these medications are important to be aware of, especially in the emergency setting, such as 
conditions related to volume depletion (hypotension, acute kidney injury), genitourinary infections, and euglycemic diabetic ketoacidosis (eDKA) [8]. As SGLT2is will be administered in a substantial number of patients in the coming years, emergency physicians should be aware of the action of these drugs and their side effects. In this review, we present practical considerations and recommendations for emergency department (ED) physicians, focusing on side effects and the management of SGLT2is in particular clinical situations.

\section{Adverse Events Related to SGLT2is}

Adverse events are categorized as very frequent (incidence of $\geq 10 \%)$, frequent $(\geq 1 \%$ and $<10 \%)$, uncommon $(\geq 0.1 \%$ and $<1 \%)$, rare $(\geq 0.01 \%$ and $<0.1 \%)$ and very rare $(<0.01 \%)$, using the Council for Organizations of Medical Sciences working group definitions, and available evidence in literature [9].

\subsection{Genitourinary Infections (Frequent to Very Frequent)}

An increased risk of genital mycotic infection is associated with SGLT2is, particularly in women (vulvovaginitis) and uncircumcised men (balanitis). Although it is the most common adverse event, most infections are mild or moderate [10]. In a meta-analysis including 36689 patients, canagliflozin, dapagliflozin, and empagliflozin were associated with higher risks of genital mycotic infections compared with placebo, with respective odd ratios (ORs) ranging from 3.64 (95\% confidence interval (CI), 2.87-4.63) for empagliflozin to 4.99 (95\% CI, 3.74-6.67) for canagliflozin [11].

Concerning the risk of urinary tract infections (UTIs), data are less consistent. Reports of pyelonephritis and complicated UTIs (urosepsis) have prompted the EMA and FDA to add warnings about an increased risk of UTIs [12]. However, in a large meta-analysis which included 110 trials, SGLT2is did not demonstrate an increased risk of UTIs [13]. These data were recently completed by a large population based cohort study which compared SGLT2is with dipeptidyl peptidase-4 (DPP-4) inhibitors and glucagon-like peptide 1 receptor agonists (GLP-1 RAs) and did not show an increased risk of UTIs associated with SGLT2is [14]. In practice, patients taking SGLT2is should be regularly informed about the importance of maintaining good local hygiene and should be educated about the signs and symptoms of genital mycotic infection and UTIs. The management of the latter is not different from usual care, with the administration of local/oral antifungal therapy or oral/intravenous anti-biotherapy as needed.

\subsection{Hypoglycemia (Frequent)}

SGLT2is inherently present a low risk of hypoglycemia because of their insulinindependent pathway of action [8]. In clinical trials, hypoglycemic events were rare and not more frequent in nondiabetic patients [1,2]. However, a concomitant use of insulin or insulin secretagogues (glinides, sulfonylureas) may increase the risk of hypoglycemia. Some authors have therefore suggested reducing the dose of sulfonylurea or glinide by $50 \%$ or the basal insulin dose by $20 \%$ when starting a SGLT2i, especially when glycated hemoglobin $(\mathrm{HbA} 1 \mathrm{C})$ at baseline is normal or when the patient has a known history of hypoglycemic events [15]. Episodes of hypoglycemia in the ED should be managed according to standard protocol, with administration of oral or intravenous glucose, and temporary withholding of the SGLT2i. The dose of other antihyperglycemic agents should subsequently be adapted.

\subsection{Volume Depletion and Acute Kidney Injury (Frequent)}

Because of its effect on osmotic diuresis, SGLT2is may cause symptomatic hypotension or dehydration (incidence of $1.2 \%$ to $1.5 \%$ ), especially in elderly patients or those already taking diuretics [16]. In patients presenting to the ED with hypotension or symptoms or signs of dehydration, temporary withholding of SGLT2is may be considered.

Regarding the risk of acute kidney injury (AKI), reports have provided conflicting conclusions. In 2016, the FDA issued warnings regarding the use of canagliflozin and 
dapagliflozin after 101 cases of AKI, some requiring dialysis [17]. Most occurred within 1 month of SGLT2i initiation. This observation has been challenged by a meta-analysis of the main clinical trials, which suggested the risk of AKI may slightly decrease via the reno-protective effects of SGLT2is [18]. In practice, a modest decrease in estimated glomerular filtration rate (eGFR) of around $3-4 \mathrm{~mL} / \mathrm{min} / 1.73 \mathrm{~m}^{2}$ is expected when starting a SGLT2i [16]. Kidney function should be assessed before treatment initiation and monitored thereafter, especially in patients with comorbidities or medications predisposing to AKI.

In patients presenting to the ED with severe AKI, SGLT2i therapy and other potentially nephrotoxic drugs should be withheld, with the correction of any electrolyte imbalance and the initiation of a standard protocol for fluid resuscitation in severe AKI resulting from circulatory hypovolemia and hemodialysis as a last resort, if needed.

\subsection{Euglycemic Diabetic Ketoacidosis (Rare)}

First reported in 2015 [19], euglycemic diabetic ketoacidosis (eDKA) associated with SGLT2is is a rare and life-threatening condition. On a physio-pathological level, it is thought that the lowered plasma glucose levels induced by glucosuria reduce the amount of insulin while increasing glucagon release [20]. The increased glucagon/insulin ratio is responsible for increased lipolysis and upregulated ketone body production ( $\beta$-hydroxybutyrate, acetoacetate), which may lead to ketoacidosis [20]. Because of the lowered plasma glucose levels related to glucosuria, ketoacidosis under SGLT2i is generally characterized by the absence of major hyperglycemia $(<250 \mathrm{mg} / \mathrm{dL})$, hence the name eDKA. Delays in diagnosis can be important because emergency physicians may not be used to ketoacidosis without severe hyperglycemia.

In clinical trials investigating patients with T2DM and those with HFrEF, SGLT2iassociated DKA was rare, with incidence rates ranging from $0.1 \%$ (empagliflozin) to 0.6/1000 patient-years (canagliflozin) [21-23]. In fact, a real-world propensity-matched study found that SGLT2is were twice more likely to cause eDKA compared with DPP4 inhibitors [24]. Using the FDA Adverse Event Reporting System (FAERS), Blau and colleagues estimated that SGLT2is increase the risk of eDKA seven-fold [25]. The reason why these observations are different from the clinical trials is unclear. However, participants in randomized trials receive closer medical attention, and this may have contributed to lowering the risk of eDKA.

EDKA should systematically be considered in patients taking SGLT2is, regardless of blood sugar levels presenting with nausea, vomiting, malaise, or abdominal pain or, in more severe cases, altered consciousness, Kussmaul breathing, or clinical signs of shock [19]. This is especially true when an additional trigger is present such as fasting, dehydration, discontinuation of insulin therapy, surgery, infections, or excessive alcohol intake [26]. EDKA may also be found in patients with T1DM, for example, when the insulin pump is defective, or in pregnant women with T1DM, as pregnancy may cause significant physiological glucosuria. Initial evaluation is similar to classic DKA and should rapidly include screening with serum $\mathrm{pH}$. If the latter is below 7.3, rapid $\beta$-hydroxybutyrate capillary checking should be considered [19].

In the ED setting, physicians may be confronted with other forms of acidosis, particularly metformin-associated lactic acidosis (MALA), which should not be confused with eDKA in patients treated by a SGLT2i and metformin at the same time. The difference between eDKA and MALA is the absence of $\beta$-hydroxybutyrate elevation in MALA, but the two disorders can coexist in theory, especially in patients with CKD [27]. Diagnostic criteria of eDKA, as defined by the American College of Endocrinology, and MALA, are presented in Table 1.

Once the diagnosis of eDKA is confirmed, SGLT2is should immediately be discontinued, and treatment should be started including fluid resuscitation, insulin with concomitant glucose infusion, careful electrolyte and glycemia monitoring, and treatment of the underlying trigger if feasible [28]. Patients taking SGLT2is should be instructed to maintain 
sufficient oral hydration and carbohydrate intake and to stop the drug in the case of emergency surgery or unexpected external severe stress events [19].

Table 1. Diagnostic criteria of euglycemic diabetic ketoacidosis and metformin-associated lactic acidosis [19,27].

\begin{tabular}{|c|c|c|}
\hline \multirow[t]{2}{*}{ Parameters } & \multicolumn{2}{|c|}{ Laboratory Values } \\
\hline & eDKA & MALA \\
\hline Arterial pH & $<7.3$ & $<7.35$ \\
\hline$\beta$-hydroxybutyrate & $\begin{array}{l}\geq 31 \mathrm{mg} / \mathrm{dL}(3.0 \mathrm{mmol} / \mathrm{L}) \text { in children } \\
\geq 40 \mathrm{mg} / \mathrm{dL}(3.8 \mathrm{mmol} / \mathrm{L}) \text { in adults }\end{array}$ & Normal \\
\hline Serum ketone & Positive & Negative \\
\hline Serum lactate & Normal or slightly elevated & $>5 \mathrm{mmol} / \mathrm{L}$ \\
\hline Anion gap & $>10 \mathrm{mmol} / \mathrm{L}$ & $>10 \mathrm{mmol} / \mathrm{L}$ \\
\hline
\end{tabular}

eDKA: Euglycemic diabetic ketoacidosis, MALA: Metformin-associated lactic acidosis.

\subsection{Necrotizing Fasciitis of the Perineum (Very Rare)}

Necrotizing fasciitis of the perineum, also known as Fournier's gangrene (FG), is a potentially fatal acute necrotic infection of the subcutaneous tissues around the genital or perianal regions. Classic risk factors for FG include hypertension, obesity, tobacco use, immunosuppression, heart failure, and T2DM [29]. Using the FAERS, Bersoff-Matcha and colleagues identified 55 cases of FG in patients receiving SGLT2is from March 2013 to January 2019 versus 19 cases in patients receiving other classes of antidiabetic therapy during a 35-year period in the U.S. population [30]. The physio-pathological explanation behind this observation is unknown.

FG should be suspected in any patient taking a SGLT2i who presents with fever along with pain, erythema, or swelling of the genital or perianal area. If diagnosed, immediate discontinuation of the SGLT2i, hospitalization, close monitoring, prompt introduction of broad-spectrum anti-biotherapy, and surgical debridement, if necessary, are required [10].

\subsection{Fractures (Unknown Incidence)}

Canagliflozin was the only SGLT2i associated with a slightly higher risk of fractures, most of them nonvertebral, in the CANVAS-Program trial, but this has not been confirmed in subsequent studies [21]. Possible explanations include a greater risk of falls due to volume depletion and a reduction in bone density [31].

\subsection{Lower Limb Amputations (Unknown Incidence)}

An increased risk of lower limb amputations (LLA) with canagliflozin, mostly toe and metatarsal ones, was reported in the CANVAS-Program trial with a HR of 1.97 (95\% CI 1.41-2.75) [21]. Although dapagliflozin and empagliflozin were not associated with the same risk in their respective clinical trials [22,23], a meta-analysis confirmed the slight increase in the risk of LLA for SGLT2is compared to controls or placebo. The mechanism by which SGLT2is might increase the risk of amputations is unknown, and whether it concerns all drugs remains controversial.

SGLT2is should be used with caution in patients with previous amputations, active cutaneous ulcers, or lower extremity artery disease [16]. In the case that peripheral arterial disease is suspected, screening should be rapidly performed.

\subsection{Stroke}

Of note, some data have reported an increased rate of stroke associated with SGLT2is, the mechanism of which is not fully understood [32]. This was, however, not confirmed in a large meta-analysis, which did not find any significant differences between dapagliflozin, canagliflozin, and empagliflozin compared with control groups [33]. 
Table 2 summarizes adverse events, their respective incidence rates, and proposed management.

Table 2. Adverse events associated with SGLT2is and proposed courses of action.

\begin{tabular}{|c|c|c|}
\hline Adverse Events & Incidence & Practical Considerations \\
\hline Mycotic genital infections & $\begin{array}{c}\text { Very frequent } \\
\text { Male } \\
\text { C: } 34.9 \text { p/1000 p-y } \\
\text { E: } 5 \% \\
\text { Female } \\
\text { C: } 68.8 \text { p } / 1000 \text { p-y } \\
\text { E: } 10 \%\end{array}$ & $\begin{array}{l}\text { - } \quad \text { Local or oral antifungal therapy } \\
\text { - } \quad \text { Reinforce patient education: Adequate hydration, good local hygiene, } \\
\text { and self-monitoring }\end{array}$ \\
\hline
\end{tabular}

Urinary tract infections

C: $40 \mathrm{p} / 1000 \mathrm{p}-\mathrm{y}$

D: $1.5 \%$

E: $1.8 \%$
- Oral or intravenous anti-biotherapy

- Reinforce patient education: Adequate hydration, good local hygiene, and self-monitoring

$\begin{array}{cc} & \text { Frequent } \\ & \text { C: } 50 \mathrm{p} / 1000 \mathrm{p}-\mathrm{y} \\ \text { Hypoglycemia } & \text { D: } 0.7 \% \\ & \text { E: } 1.3 \%\end{array}$

E: $1.3 \%$
- Administration of oral or intravenous glucose

- Withholding of SGLT2i

- Hospitalization if required

- When restarting antidiabetic therapy, the dose of other antihyperglycemic agents should be adapted: Sulfonylurea or glinide by at least $50 \%$ and basal insulin by at least $20 \%$ $\begin{array}{cc}\text { Frequent } \\ \text { Hypotension } & \text { C: } 26 \mathrm{p} / 1000 \text { p-y } \\ \text { E: } 5.1 \%\end{array}$
- Oral or intravenous fluid administration

- Hospitalization if required

- $\quad$ Adapt the dose of antihypertensive treatments

- Inform patients to maintain adequate oral hydration

- Treat concomitant conditions (diarrhea ... )

- If severe condition related to volume depletion, consider temporary withholding of SGLT2i

Frequent
Acute kidney injury
C: 3 p $/ 1000$ p-y
D: $1.5 \%$
E: $1.0 \%$

- Oral or intravenous fluid administration

- Hospitalization if required

- $\quad$ Adapt the dose of other medications that may cause AKI (nonsteroidal anti-inflammatory drugs, diuretics, renin-angiotensin-aldosterone inhibitors)

- Inform patients to maintain adequate oral hydration

- Treat concomitant conditions such as diarrhea, if indicated

- $\quad$ If severe AKI, consider temporary withholding of SGLT2i

$\begin{array}{cc} & \text { Rare } \\ \text { Diabetic ketoacidosis } & \text { C: } 0.6 \mathrm{p} / 1000 \mathrm{p}-\mathrm{y} \\ \text { D: } 0.3 \% \\ \text { E: } 0.1 \%\end{array}$

Fournier's gangrene

\section{Very rare}

C, D and $\mathrm{E}:<0.1 \%$
- Hospitalization in intensive care unit

- $\quad$ SGLT2i discontinuation

- $\quad$ Fluid resuscitation, intravenous insulin and glucose continuous infusion, careful electrolyte and glycemia monitoring (target: 8-11 mmol/L)

- $\quad$ Treatment of the underlying trigger

- Inform patients to avoid precipitating factors: Fasting, dehydration, discontinuation of insulin therapy, surgery, infections, or excessive alcohol intake 
Table 2. Cont.

\begin{tabular}{|c|c|c|}
\hline Adverse Events & Incidence & Practical Considerations \\
\hline Fractures & C:15.4 p/1000 p-y & $\begin{array}{ll}\text { - } & \text { Conservative or surgical management } \\
\text { - } & \text { Instruct patients to maintain adequate calcium uptake } \\
\text { - } & \text { Consider osteoporosis screening }\end{array}$ \\
\hline Lower limb amputations & $\begin{array}{c}C: 6.4 \mathrm{p} / 1000 \mathrm{p}-\mathrm{y} \\
\text { D: } 1.4 \%\end{array}$ & $\begin{array}{ll}\text { - } & \text { Use SGLT2is with caution in patients with previous amputations or } \\
\text { - } & \text { peripheral artery disease } \\
\text { - } & \text { Rem threshold to screen for peripheral arterial disease } \\
& \text { Remind patients to perform regular foot exams }\end{array}$ \\
\hline
\end{tabular}

AKI: Acute kidney injury. Incidence rates of side effects are based on the CANVAS-Program [21], DECLARE-TIMI 58 [22], and EMPAREGOUTCOME [23] trials. They are expressed as number of patients for 1000 patient-years ( $\mathrm{p} / \mathrm{P}$-y=patients / 1000 patient-years) for canagliflozin (C), as percentages for dapagliflozin (D) and empagliflozin (E). SLGT2i: Sodium-glucose cotransporter 2 inhibitor.

\section{Use of SGLT2is in Particular Clinical Situations}

In general, it is advised to discontinue SGLT2is in patients undergoing urgent surgery or hospitalized with any acute serious medical condition (e.g., infections, stroke, acute kidney or liver dysfunction) because of the risk of eDKA. In case of scheduled surgery, the FDA recommends discontinuing SGLT2is $72 \mathrm{~h}$ prior to intervention ( $96 \mathrm{~h}$ for ertugliflozin) [34].

However, in certain acute clinical situations, discontinuing the SGLT2i may be debatable. In the following section, we discuss the management of patients taking SGLT2is at baseline, presenting to the ED in four different clinical situations.

\subsection{Acute Heart Failure}

It is estimated that more than 26 million people suffer from heart failure worldwide, with up to $77 \%$ presenting at least once to the ED with decompensated acute heart failure (AHF) [35]. As such, the ED serves as the portal of entry for the majority of AHF admissions, and managing SGLT2is in patients presenting with AHF is already and will become an increasingly frequent challenge in the coming years. The role and use of SGLT2is in AHF has not yet been investigated in large-scale trials. However, in a randomized pilot study comparing empagliflozin against placebo in 80 patients with AHF (with and without T2DM), Damman et al. showed that empagliflozin was associated with a greater urinary output and a reduction in a combined outcome of worsening HF, death, and/or rehospitalization for AHF at 60 days [36]. In a position statement from the Heart Failure Association of the European Society of Cardiology, SGLT2is are considered as third-line diuretic treatment with added loop diuretics, after thiazides or acetazolamide/amiloride, based on their natriuretic and osmotic diuretic effect [37]. In view of these data, in an AHF patient with a SGLT2i at baseline, it seems reasonable to continue the treatment provided the patient does not have any sign of hemodynamic instability and does not present contraindications or side effects as described above (e.g., eDKA, AKI, hypotension). In patients with "wet" (congestive) AHF, temporarily increasing the dose of the SGLT2i (if possible) may be an option if additional diuretic effect is necessary after the initiation of standard diuretic therapy. In patients with "dry" AHF, keeping the same dose of SGLT2i is an option.

\subsection{Atrial Fibrillation with Rapid Ventricular Response}

Literature regarding the management of SGLT2is in the setting of patients presenting with atrial fibrillation (AF) with rapid ventricular response is very scarce. However, in authors' opinion, the decision to discontinue or not the SGLT2i primarily depends on the hemodynamic tolerance of the AF and the volume status. In patients with hemodynamic instability (symptomatic hypotension, cardiogenic shock) and in those presenting with hypovolemia based on clinical and biological evaluation, it seems reasonable to withhold 
the SGLT2i in addition to general AF management (initiation of rhythm or rate control, fluid resuscitation, and initiation of anticoagulation, if needed).

\subsection{Acute Diabetes Decompensation}

Acute diabetes decompensation is another commonly encountered condition in the ED and includes hyperosmolar hyperglycemic state (HHS), eDKA, and marked hyperglycemia without hyperosmolar or ketosis conditions. Although historically considered as two distinct clinical entities, HHS and eDKA share the same basic pathophysiologic mechanisms: Significant insulin deficiency and increased concentration of counterregulatory hormones such as glucagon, catecholamines, cortisol, and growth hormone. Furthermore, similar to patients with eDKA, those with HHS frequently may present with signs of dehydration, dry mucous membranes and poor skin turgor, or hypotension [38]. Although no clear recommendation regarding HHS and the use of SGLT2is exists, in patients with a SGLT2i at baseline presenting to the ED with HHS, it seems reasonable to initiate standard HHS treatment: fluid resuscitation, insulin infusion, careful electrolyte and glycemia monitoring, and treatment of the underlying cause.

\subsection{Gout Attack}

SGLT2is have been shown to consistently reduce uric acid concentrations via increased urinary uric acid excretion by approximately $3545 \mu \mathrm{mol} / \mathrm{L}$ to $45 \mu \mathrm{mol} / \mathrm{L}(0.60-0.75 \mathrm{mg} / \mathrm{dL})$. The lowering of uric acid by SGLT2is may partly explain the beneficial CV and renal effects associated with this class of treatments [8]. When compared with traditional hypouricemic treatments (xanthine oxidase inhibitors), their mode of action is different and potentially complementary [39]. Whether SGLT2is may be useful in the management of hyperuricemia is still unknown, but it seems reasonable, in patients with a SGLT2i at baseline and presenting with a gout attack, to continue the SGLT2i, providing they do not have any condition which might put them at risk of developing eDKA (AKI, significant inflammatory syndrome....).

Table 3 summarizes the management of SGLT2is associated with the four precited clinical situations.

Table 3. Proposed management of patients taking SGLT2is at baseline in the case of acute heart failure, atrial fibrillation with rapid ventricular response, acute diabetes decompensation, and gout attack.

\begin{tabular}{|c|c|}
\hline Conditions & Proposed Course of Actions \\
\hline $\begin{array}{l}\text { Any planned or unplanned surgery } \\
\text { Any acute serious medical condition with need of } \\
\text { hospitalization, apart from the ones discussed below }\end{array}$ & Discontinue SGLT2i \\
\hline \multirow{4}{*}{ Acute heart failure } & Hemodynamically stable patients \\
\hline & $\begin{array}{l}\text { - "Wet" AHF: Continue SGLT2i at the same or at an increased dosage, } \\
\text { along with standard diuretic therapy } \\
\text { - } \quad \text { Dry" AHF: Continue SGLT2i at the same dosage }\end{array}$ \\
\hline & Hemodynamically unstable patients \\
\hline & - $\quad$ Discontinue SGLT2i \\
\hline
\end{tabular}

\section{Hemodynamically unstable patients}

- $\quad$ Discontinue SGLT2i

Atrial fibrillation with rapid ventricular response

Hemodynamically stable patients

- Hypovolemic patients: Discontinue SGLT2i

- $\quad$ Euvolemic or hypervolemic patients: Continue SGLT2i at the same dosage 
Table 3. Cont.

\section{Conditions}

Acute diabetes decompensation
Proposed Course of Actions

Thoroughly evaluate the patient for eDKA or HHS

In case of eDKA or HHS

- Immediately discontinue SGLT2i

- $\quad$ Associated measures: fluid resuscitation, insulin infusion, careful electrolyte and glycemia monitoring, treatment of the underlying trigger

In case of isolated hyperglycemia

- $\quad$ Switching to insulin therapy may be preferable

\section{Gout attack} SGLT2i may be continued

AHF: Acute heart failure, eDKA: Euglycemic diabetic ketoacidosis, HHS: Hyperosmolar hyperglycemic state, SLGT2i: Sodium-glucose cotransporter 2 inhibitor.

\subsection{Pregnancy}

No well-controlled studies of SGLT2is have been performed in the context of pregnancy. According to animal studies, these drugs may affect renal development. Therefore, SGLT2is should be used in pregnant women only if the benefits justify the risk to the fetus [40].

\section{Conclusions}

SGLT2is are antidiabetic drugs for which indications are currently rapidly expanding, as new data show beneficial effects not only in patients with T2DM, but also in those with HFrEF and CKD, regardless of the diabetes status. Overall, they are well-tolerated treatments, with mild genital mycotic infections and volume depletion being the most common adverse events. However, as the number of patients taking SGLT2is increases, emergency physicians may be faced with rarer adverse events, some of which, if left unrecognized, could be life-threatening. The risk of adverse events may be reduced by careful patient information and education about self-monitoring. Although it is advised to discontinue SGLT2is in patients undergoing urgent surgery or hospitalized with any acute serious medical condition, in certain acute clinical situations, such as acute heart failure, this may be debatable, as SGLT2 inhibition may actually yield beneficial effects.

Author Contributions: H.L. (Henri Lu), H.L. (Hortense Lu). and R.H. were responsible for the article concept. H.L. (Henri Lu) and H.L. (Hortense Lu) drafted the first version of the article. All authors were responsible for content analysis, assisted in drafting the article, and made critical revisions for important intellectual content. R.H. takes responsibility for the paper as a whole. All authors have read and agreed to the published version of the manuscript.

Funding: This article received no external funding.

Institutional Review Board Statement: Not applicable.

Informed Consent Statement: Not applicable.

Conflicts of Interest: H.L. (Henri Lu), H.L. (Hortense Lu), C.K., P.-N.C., M.M., J.M., O.M. and R.H. do not have any conflict of interest in relation with this article. A.W. reports consulting honoraria from Boehringer Ingelheim, Astra Zeneca and Janssen. A.Z. reports consulting honoraria from Boehringer Ingelheim, Astra Zeneca and Mundipharma. A.Z. also participated in clinical trials for Boehringer Ingelheim. P.M. has participated to meetings organized by Boehringer Ingelheim, whose honoraria have been entirely paid to a private research foundation of the Cardiology Service of the University Hospitals of Geneva (GeCor foundation). The funders had no role in the writing or in the decision to publish the manuscript. 


\section{References}

1. McMurray, J.J.V.; Solomon, S.D.; Inzucchi, S.E.; Køber, L.; Kosiborod, M.N.; Martinez, F.A.; Ponikowski, P.; Sabatine, M.S.; Anand, I.S.; Bělohlávek, J.; et al. Dapagliflozin in Patients with Heart Failure and Reduced Ejection Fraction. N. Engl. J. Med. 2019, 381, 1995-2008. [CrossRef]

2. Packer, M.; Anker, S.D.; Butler, J.; Filippatos, G.; Pocock, S.J.; Carson, P.; Januzzi, J.; Verma, S.; Tsuitsui, H.; Brueckmann, M.; et al. Cardiovascular and Renal Outcomes with Empagliflozin in Heart Failure. N. Engl. J. Med. 2020, 383, 1413-1424. [CrossRef]

3. Heerspink, H.J.L.; Stefánsson, B.V.; Correa-Rotter, R.; Chertow, G.M.; Greene, T.; Hou, F.-F.; Mann, J.F.E.; McMurray, J.J.V.; Lindberg, M.; Rossing, P.; et al. Dapagliflozin in Patients with Chronic Kidney Disease. N. Engl. J. Med. 2020, 383, 1436-1446. [CrossRef]

4. Cosentino, F.; Grant, P.J.; Aboyans, V.; Bailey, C.J.; Ceriello, A.; Delgado, V.; Federici, M.; Filippatos, G.; Grobbee, D.E.; Hansen, T.B.; et al. 2019 ESC Guidelines on diabetes, pre-diabetes, and cardiovascular diseases developed in collaboration with the EASD. Eur. Heart J. 2020, 41, 255-323. [CrossRef]

5. American Diabetes Association. 9. Pharmacologic approaches to glycemic treatment: Standards of Medical Care in Diabetes-2020. Diabetes Care 2020, 43 (Suppl. 1), S98-S110. [CrossRef]

6. U.S. Food and Drug Administration. FDA Approves New Treatment for a Type of Heart Failure. 2020. Available online: https://www.fda.gov/news-events/press-announcements/fda-approves-new-treatment-type-heart-failure (accessed on 13 December 2020).

7. European Medicines Agency. Forxiga is indicated in adults for the treatment of symptomatic chronic heart failure with reduced ejection fraction. 2020. Available online: https://www.ema.europa.eu/en/documents/product-information/forxiga-eparproduct-information_en.pdf (accessed on 16 January 2020).

8. Lu, H.; Meyer, P.; Hullin, R. Use of SGLT2 inhibtors in cardiovascular diseases: Why, when and how? A narrative literature review. Swiss. Med. Wkly. 2020, 150, w20341. [PubMed]

9. Council for International Organizations of Medical Sciences. Guidelines for Preparing Core Clinical-Safety Information on Drugs: Report of CIOMS Working Groups III and V; Including New Proposals for Investigator's Brochures; CIOMS: Geneva, Switzerland, 2001; Volume 98.

10. McGill, J.B.; Subramanian, S. Safety of Sodium-Glucose Co-Transporter 2 Inhibitors. Am. J. Med. 2019, 132, 14. [CrossRef]

11. Li, D.; Wang, T.; Shen, S.; Fang, Z.; Dong, Y.; Tang, H. Urinary tract and genital infections in patients with type 2 diabetes treated with sodium-glucose co-transporter 2 inhibitors: A meta-analysis of randomized controlled trials. Diabetes Obes. Metab. 2017, 19, 348. [CrossRef] [PubMed]

12. Sarafidis, P.A.; Ortiz, A. The risk for urinary tract infections with sodium-glucose cotransporter 2 inhibitors: No longer a cause of concern? Clin. Kidney J. 2020, 13, 24-26. [CrossRef] [PubMed]

13. Donnan, J.R.; Grandy, C.A.; Chibrikov, E.; Marra, C.A.; Aubrey-Bassler, K.; Johnston, K.; Swab, M.; Hache, J.; Curnew, D.; Nguyen, H.; et al. Comparative safety of the sodium glucose co-transporter 2 (SGLT2) inhibitors: A systematic review and meta-analysis. BMJ Open 2019, 9, e022577. [CrossRef]

14. Dave, C.V.; Schneeweiss, S.; Kim, D.; Fralick, M.; Tong, A.; Patorno, E. Sodium-Glucose Cotransporter-2 Inhibitors and the Risk for Severe Urinary Tract Infections: A Population-Based Cohort Study. Ann. Intern. Med. 2019, 171, 248-256. [CrossRef] [PubMed]

15. Das, S.R.; Everett, B.M.; Birtcher, K.K.; Brown, J.M.; Cefalu, W.T.; Januzzi, J.L.; Kalyani, R.R.; Kosiborod, M.; Magwire, M.L.; Morris, P.B.; et al. 2018 ACC Expert Consensus Decision Pathway on Novel Therapies for Cardiovascular Risk Reduction in Patients With Type 2 Diabetes and Atherosclerotic Cardiovascular Disease. J. Am. Coll. Cardiol. 2018, 72, 3200-3223. [CrossRef] [PubMed]

16. Vardeny, O.; Vaduganathan, M. Practical Guide to Prescribing Sodium-Glucose Cotransporter 2 Inhibitors for Cardiologists. JACC Heart Fail 2019, 7, 169-172. [CrossRef]

17. U.S. Food and Drug Administration. FDA Drug Safety Communication: FDA Strengthens Kidney Warnings for Diabetes Medicines Canagliflozin (Invokana, Invokamet) and Dapagliflozin (Farxiga, Xigduo XR). 2016. Available online: https:/ /www. fda.gov / Drugs / DrugSafety / ucm505860.htm (accessed on 2 March 2020).

18. Neuen, B.L.; Young, T.; Heerspink, H.J.L.; Neal, B.; Perkovic, V.; Billot, L.; Mahaffey, K.W.; Charytan, D.M.; Wheeler, D.C.; Arnott, C.; et al. SGLT2 inhibitors for the prevention of kidney failure in patients with type 2 diabetes: A systematic review and meta-analysis. Lancet Diabetes Endocrinol. 2019, 7, 845-854. [CrossRef]

19. Handelsman, Y.; Henry, R.R.; Bloomgarden, Z.T.; Dagogo-Jack, S.; DeFronzo, R.A.; Einhorn, D.; Ferrannini, E.; Fonseca, V.A.; Garber, A.J.; Grunberger, G.; et al. American association of clinical endocrinologists and American College of Endocrinology position statement on the association of SGLT-2 inhibitors and diabetic ketoacidosis. Endocr. Pract. 2016, 22, 753-762. [CrossRef] [PubMed]

20. Goldenberg, R.M.; Berard, L.D.; Cheng, A.Y.Y.; Gilbert, J.D.; Verma, S.; Woo, V.C.; Yale, J.-F. SGLT2 Inhibitor-associated Diabetic Ketoacidosis: Clinical Review and Recommendations for Prevention and Diagnosis. Clin. Ther. 2016, 38, 2654-2664. [CrossRef]

21. Neal, B.; Perkovic, V.; Mahaffey, K.W.; de Zeeuw, D.; Fulcher, G.; Erondu, N.; Shaw, W.; Law, G.; Desai, M.; Matthews, D.R.; et al. Canagliflozin and Cardiovascular and Renal Events in Type 2 Diabetes. N. Engl. J. Med. 2017, 377, 644-657. [CrossRef]

22. Wiviott, S.D.; Raz, I.; Bonaca, M.P.; Mosenzon, O.; Kato, E.T.; Cahn, A.; Silverman, M.G.; Zelniker, T.A.; Kuder, J.F.; Murphy, S.A.; et al. Dapagliflozin and Cardiovascular Outcomes in Type 2 Diabetes. N. Engl. J. Med. 2019, 380, 347-357. [CrossRef] 
23. Zinman, B.; Wanner, C.; Lachin, J.M.; Fitchett, D.; Bluhmki, E.; Hantel, S.; Mattheus, M.; Devins, T.; Johansen, O.E.; Woerle, H.J.; et al. Empagliflozin, Cardiovascular Outcomes, and Mortality in Type 2 Diabetes. N. Engl. J. Med. 2015, 373, 2117-2128. [CrossRef]

24. Fralick, M.; Schneeweiss, S.; Patorno, E. Risk of Diabetic Ketoacidosis after Initiation of an SGLT2 Inhibitor. N. Engl. J. Med. 2017, 376, 2300-2302. [CrossRef]

25. Blau, J.E.; Tella, S.H.; Taylor, S.I.; Rother, K.I. Ketoacidosis associated with SGLT2 inhibitor treatment: Analysis of FAERS data. Diabetes Metab. Res. Rev. 2017, 33, e2924. [CrossRef]

26. Dizon, S.; Keely, E.J.; Malcolm, J.; Arnaout, A. Insights into the recognition and management of SGLT2-inhibitor-associated ketoacidosis: It's not just euglycemic diabetic ketoacidosis. Can. J. Diabetes 2017, 41, 499-503. [CrossRef]

27. Lalau, J.D.; Kajbaf, F.; Protti, A.; Christensen, M.M.; De Broe, M.E.; Wiernsperger, N. Metformin-lactic acidosis (MALA): Moving towards a new paradigm. Diabetes Obes. Metab. 2017, 19, 1502-1512. [CrossRef] [PubMed]

28. Brown, F.; McColl, T. Euglycemic Diabetic Ketoacidosis Secondary to Dapagliflozin Use: A Case Report. J. Emerg. Med. 2018, 54, 109-111. [CrossRef] [PubMed]

29. Voelzke, B.B.; Hagedorn, J.C. Presentation and diagnosis of Fournier gangrene. Urology 2018, 114, 8-13. [CrossRef]

30. Bersoff-Matcha, S.J.; Chamberlain, C.; Cao, C.; Kortepeter, C.; Chong, W.H. Fournier Gangrene Associated With Sodium-Glucose Cotransporter-2 Inhibitors: A Review of Spontaneous Postmarketing Cases. Ann. Intern. Med. 2019, 170, 764-769. [CrossRef]

31. Opingari, E.; Partridge, A.C.R.; Verma, S.; Bajaj, H.S. SGLT2 inhibitors: Practical considerations and recommendations for cardiologists. Curr. Opin. Cardiol. 2018, 33, 676-682. [CrossRef] [PubMed]

32. Wu, J.H.; Foote, C.; Blomster, J.; Toyama, T.; Perkovic, V.; Sundström, J.; Neal, B. Effects of sodium-glucose cotransporter-2 inhibitors on cardiovascular events, death and major safety outcomes in adults with type 2 diabetes: A systematic review and meta-analysis. Lancet Diabetes Endocrinol. 2016, 4, 411-419. [CrossRef]

33. Guo, M.; Ding, J.; Li, J.; Wang, J.; Zhang, T.; Liu, C.; Huang, W.; Long, Y.; Gao, C.; Xu, Y. SGLT2 inhibitors and risk of stroke in patients with type 2 diabetes: A systematic review and meta-analysis. Diabetes Obes. Metab. 2018, 20, 1977-1982. [CrossRef] [PubMed]

34. U.S. Food and Drug Administration. FDA Revises Labels of SGLT2 Inhibitors for Diabetes to Include Warnings about Too Much Acid in the Blood and Serious Urinary Tract Infections. 2020. Available online: https://www.fda.gov/drugs/drug-safety-andavailability / fda-revises-labels-sglt2-inhibitors-diabetes-include-warnings-about-too-much-acid-blood-and-serious (accessed on 3 March 2021).

35. Adams, K.F., Jr.; Fonarow, G.C.; Emerman, C.L.; LeJemtel, T.H.; Costanzo, M.R.; Abraham, W.T.; Berkowitz, R.L.; Galvao, M.; Horton, D.P.; for the ADHERE Scientific Advisory Committee and Investigators. Characteristics and outcomes of patients hospitalized for heart failure in the United States: Rationale, design, and preliminary observations from the first 100,000 cases in the Acute Decompensated Heart Failure National Registry (ADHERE). Am. Heart J. 2005, 149, 209-216. [CrossRef]

36. Damman, K.; Beusekamp, J.C.; Boorsma, E.M.; Swart, H.P.; Smilde, T.D.J.; Elvan, A.; van Eck, J.W.M.; Heerspink, H.J.L.; Voors, A.A. Randomized, double-blind, placebo-controlled, multicentre pilot study on the effects of empagliflozin on clinical outcomes in patients with acute decompensated heart failure (EMPA-RESPONSE-AHF). Eur. J. Heart Fail 2020, 22, 713-722. [CrossRef] [PubMed]

37. Mullens, W.; Damman, K.; Harjola, V.-P.; Mebazaa, A.; Brunner-La Rocca, H.-P.; Martens, P.; Testani, J.M.; Tang, W.H.W.; Orso, F.; Rossignol, P.; et al. The use of diuretics in heart failure with congestion-A position statement from the Heart Failure Association of the European Society of Cardiology. Eur. J. Heart Fail 2019, 19, 137-155. [CrossRef] [PubMed]

38. Umpierrez, G.E.; Murphy, M.B.; Kitabchi, A.E. Diabetic Ketoacidosis and Hyperglycemic Hyperosmolar Syndrome. Diabetes Spectr. 2002, 15, 28-36. [CrossRef]

39. Bailey, C.J. Uric acid and the cardio-renal effects of SGLT2 inhibitors. Diabetes Obes. Metab. 2019, 21, 1291-1298. [CrossRef] [PubMed]

40. Mosley, H.F., II; Smith, L.; Everton, E.; Fellner, C. Sodium-Glucose linked Transporter 2 (SGLT2) Inhibitors in the Management of Type-2 Diabetes: A drug Class Overview. Pharm. Ther. 2015, 40, 451-462. 